\title{
An Extragastrointestinal Stromal Tumor in the Omentum With Peritoneal Seeding Mimicking an Appendiceal Mucinous Cancer With Carcinomatosis
}

\author{
Jeonghyun Kang, Tae Joo Jeon ${ }^{1}$, Sun Och Yoon², Kang Young Lee, Seung-Kook Sohn \\ Departments of Surgery, ${ }^{1}$ Nuclear Medicine, and ${ }^{2}$ Pathology, Yonsei University College of Medicine, Seoul, Korea
}

Gastrointestinal stromal tumors (GISTs) are the most common mesenchymal tumors of the gastrointestinal tract. These tumors tend to present most frequently in the stomach, followed by the small intestine. GISTs can also arise from the omentum, retroperitoneum, mesentery, or pleura and are termed extragastrointestinal stromal tumors (EGISTs) when they do so. EGISTs arising from the omentum are very rare. Due to the limited incidence of EGISTs in the omentum, the diagnostic criteria are not well established, and making a correct diagnosis may be difficult. In this report, we present a case of an EGIST of the omentum with peritoneal metastasis that was initially suspected to be an appendiceal mucinous carcinoma with carcinomatosis on positron emission tomography/computed tomography imaging.

Keywords: Gastrointestinal stromal tumors; Positron emission tomography

\section{INTRODUCTION}

Gastrointestinal stromal tumors (GISTs) are rare and account for only $2.0 \%$ of all gastrointestinal neoplasms [1]. GISTs are the most common mesenchymal tumors of the gastrointestinal tract. The most commonly involved site is the stomach, followed by the small intestine [2]. GISTs can be identified as a distinct subset by using c-kit (CD 117) which is positive in almost all GISTs [3]. Histologically, GISTs are known to originate from precursors of the interstitial cells of Cajal [4]. In rare examples, GISTs can also arise from the omentum, retroperitoneum, mesentery, or pleura, and they are categorized as extragastrointestinal stromal tumors (EGISTs) [5]. EGISTs of the omentum are rare. Due to the limited incidence of EGISTs in the omentum, an accurate diagnosis

Received: August 4, 2013 • Accepted: November 24, 2013

Correspondence to: Tae Joo Jeon, M.D.

Department of Nuclear Medicine, Gangnam Severance Hospital, Yonsei

University College of Medicine, 211 Eonju-ro, Gangnam-gu, Seoul 135-720, Korea

Tel: +82-2-2019-3740, Fax: +82-2-3462-5472

E-mail: tjeonnm@yuhs.ac

(c) 2014 The Korean Society of Coloproctology

This is an open-access article distributed under the terms of the Creative Commons Attribution NonCommercial License (http://creativecommons.org/licenses/by-nc/3.0) which permits unrestricted noncommercial use, distribution, and reproduction in any medium, provided the original work is properly cited. might be somewhat difficult to make preoperatively. We experienced a case of an EGIST of the omentum with peritoneal metastasis that was initially suspected to be an appendiceal mucinous carcinoma with carcinomatosis.

\section{CASE REPORT}

A 54-year-old male was admitted to the emergency room with right lower quadrant (RLQ) abdominal pain. He had a history of hypertension and diabetes mellitus and was being treated at an outpatient clinic with regular medications. In addition, he had chronic kidney disease. On physical examination in the emergency room, his body temperature was $36.8^{\circ} \mathrm{C}$, his heart rate was 86 per minute, his blood pressure was $186 / 113 \mathrm{mmHg}$, and his respiratory rate was 18 per minute. Diffuse lower abdominal pain of the right-abdomen was noted. On laboratory examination, the white blood cell count was 6,490 cells/ $\mathrm{mm}^{3}$ (segment form, 57.6\%) and the blood chemistry was abnormal: blood urea nitrogen (BUN), $29.8 \mathrm{mg} / \mathrm{dL}$; creatinine (Cr), $1.68 \mathrm{mg} / \mathrm{dL}$; lipase, 113U/L; and troponin T, $0.035 \mathrm{mcg} / \mathrm{L}$. Tumor markers, such as carcinoembryonic antigen and carbohydrate antigen 19-9, were within normal ranges.

Because of the patient's history of chronic renal disease and the elevated BUN/Cr level at initial examination, an abdominopelvic 


\section{Coloporocilogy}

computed tomography (CT) was performed without contrast media. The CT revealed heterogeneous soft tissue lesions in the RLQ with an embedded appendix and multiple nodules in the omentum, mesentery, and pelvis. However, the results were inconclusive due to the lack of contrast enhancement. On a contrast-enhanced abdominopelvic CT performed after the recovery of renal function with hydration, loculated cystic and solid masses measuring up to $7 \mathrm{~cm}$ were noted in the RLQ, and the appendix was invisible. Multiple seeding nodules were observed in the greater omentum, subhepatic space, bilateral paracolic, mesentery, pelvis, and rectal shelf, with a moderate number of malignant ascites. However, no gross primary mass was seen in the gastrointestinal tract or the pancreas.

On a F-18 fluorodeoxyglucose positron emission tomography/ computed tomography (FDG PET/CT) scan to evaluate systemic dissemination, an approximately $7.3-\mathrm{cm}$ hypermetabolic mass with a focal cystic portion was seen in the retrocecal area, and other large hypermetabolic masses with cystic portions were also seen in the pelvic cavity. Multiple hypermetabolic foci and omental cakes were noted along the peritoneum and the greater omentum, with a large number of ascites (Fig. 1). Accordingly, the most probable preoperative radiologic diagnosis was an appendiceal mucinous carcinoma with peritoneal seeding.

Surgery with curative intent was performed, and numerous cystic nodules were found in the whole peritoneum, but mainly in greater omentum. The appendix was grossly normal. A curative $\mathrm{R} 0$ resection was not possible due to the dissemination of multiple small cystic masses in the whole abdominal cavity. Only a partial resection of the greater omentum, the excision of a large cystic mass located in the RLQ area, and an appendectomy were performed with a palliative aim (Fig. 2).

The pathologic diagnosis was a malignant GIST, and mitosis was up to 7/10 high power fields, with necrosis showing epithelioid features, hemorrhage and cystic degeneration. Immunohistochemically, the tumor cells were positive for C-kit, vimentin, synaptophysin and S-100, but negative for CD34, $\alpha$-smooth muscle
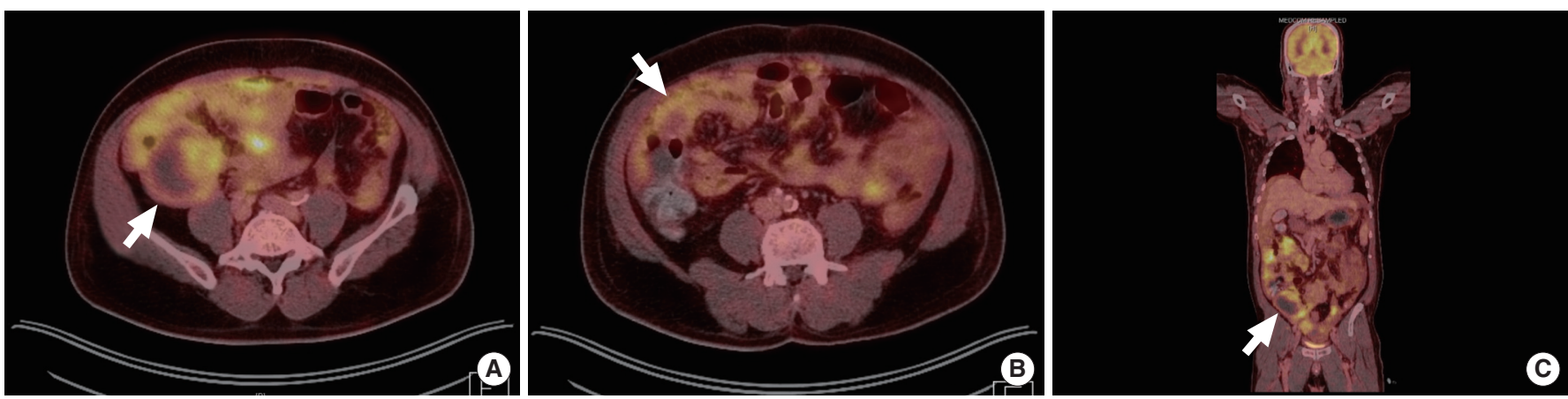

Fig. 1. Positron emission tomography/computed tomography findings. (A) A hypermetabolic mass with a focal cystic portion in the retrocecal area (white arrow). (B, C) Multiple hypermetabolic foci and omental cakes along the peritoneum and greater omentum (white arrow, respectively).

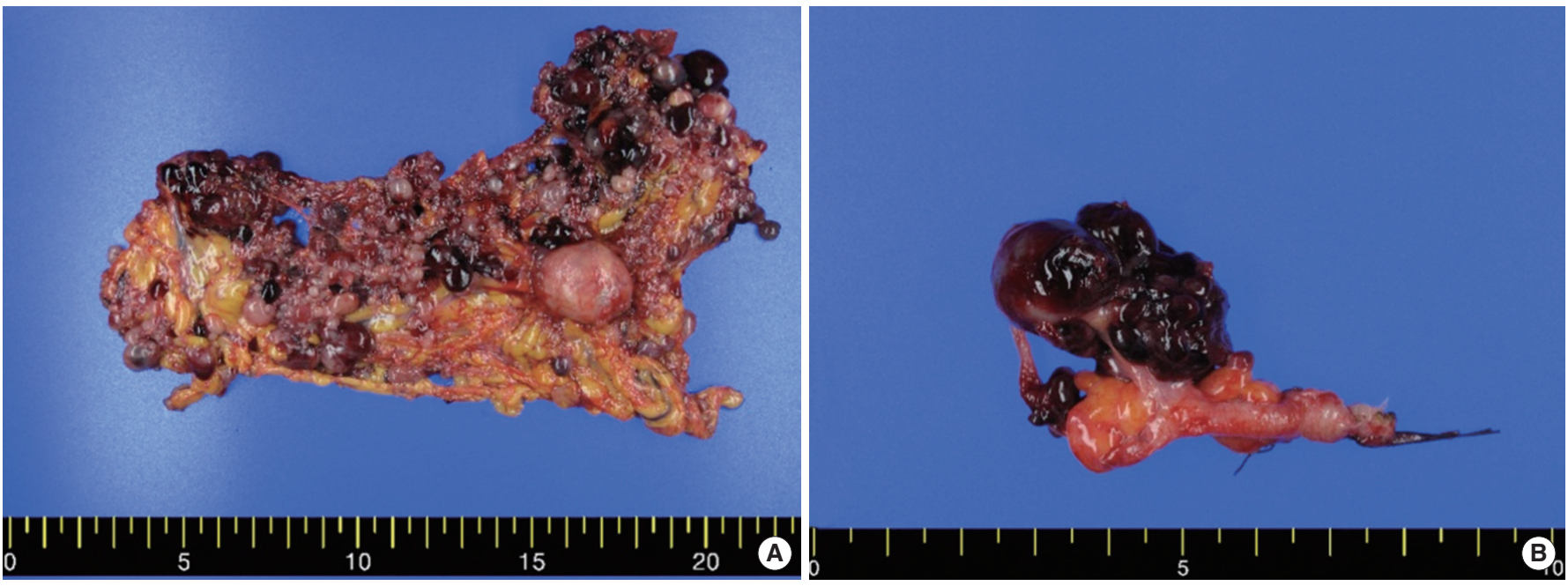

Fig. 2. Surgical specimen. (A) Variously-sized multiple grape-like nodules were detected in the greater omentum. (B) The appendix with peritoneal seeding nodules. The appendix itself showed no abnormal findings. 

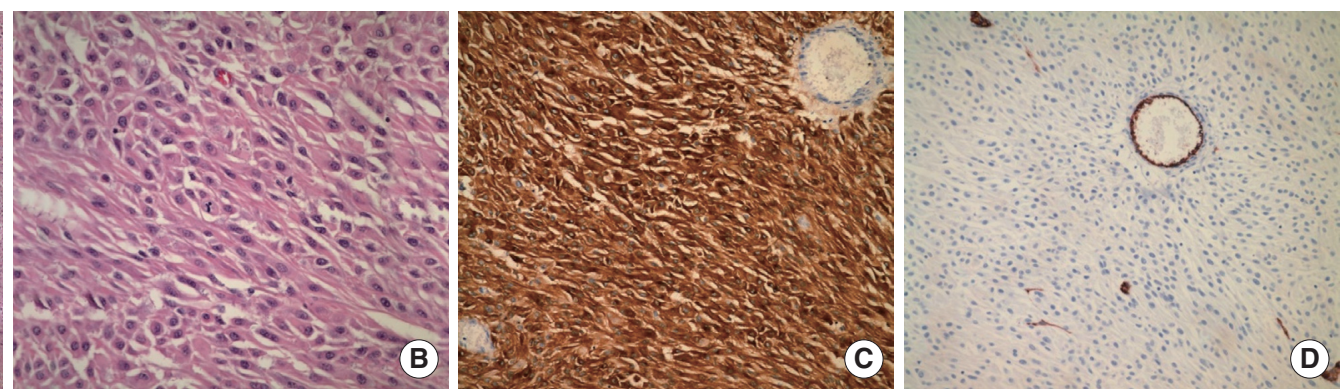

Fig. 3. Pathologic examinations. (A) Partly spindling and predominantly epitheliod tumor cells, hematoxylin-eosin stain ( $\times 100)$. (B) Epithelioid tumor cells and tripolar mitosis, hematoxylin-eosin stain $(\times 400)$. (C) C-kit diffuse cytoplasmic expression $(\times 200)$. (D) CD34 negative in tumor cells, only positive in the endothelial cells of blood vessels $(\times 200)$.

actin (SMA), and myeloperoxidase (Fig. 3).

After the diagnosis of a malignant GIST, additional studies, such as a small bowel series, esophagogastroduodenoscopy, and colonoscopy, were done to find the primary site. However, no primary suspected mass or abnormal finding was seen on those examinations. Imatinib mesylate (Gleevec, Novartis, Basel, Switzerland) was started for further adjuvant treatment.

\section{DISCUSSION}

In this case, we found innumerable grape-like omental nodules during exploration [5]. Although locating the epicenter of the primary large tumor was difficult, we believe that the gastrointestinal tract might not have been the origin of the disease because we could not find any abnormal manifestations in the bowel walls on esophagogastroduodenoscopy, colonoscopy, or the small bowel series. In addition, pathologic examination revealed that the cystic masses were surrounded by omental tissues, not by gastrointestinal wall tissue. Two masses in the RLQ of the abdomen, which were detected on the preoperative PET/CT, were not derived from any kind of gastrointestinal tract, mesentery or retroperitoneum. For this reason, we determined our patient as having an EGIST arising from the omentum.

Primary GISTs have been reported to be hypervascular and heterogeneous because of necrosis, hemorrhage, and cystic degeneration [6]. CT is a useful diagnostic tool in the localization of a GIST. Typical CT findings of a GIST have been reported to include central fluid attenuation and heterogeneity of the soft tissue attenuation [7]. The CT findings of a primary GIST in the omentum and mesentery are known to be well-marginated, lobulated masses containing large areas of low attenuation without central gas [8]. However, the CT features of GISTs can be variable, depending on the tumor's location, size, and malignant potential [9].

$\mathrm{PET} / \mathrm{CT}$ is an emerging tool for treatment response evaluation, as well as diagnosis, in various tumors and has been used in estimating treatment response in GIST patients $[10,11]$. In addition, PET/CT is known to be able to predict the malignant potential of a GIST [12]. As far as we know, no typical findings of EGISTs in
PET-CT examinations have been reported so far.

In the current case, the preoperative diagnosis was an appendiceal mucinous neoplasm with carcinomatosis. In evaluating disseminated mucinous carcinomas, PET imaging has inherent limitations because hypocellular tumors usually have low FDG uptake and that leads to a low sensitivity [13]. PET results have been reported to be positive in $35 \%$ of all cases and even in patients with high-grade disseminated cancer of the appendix; the sensitivity was only $41 \%$ [14]. In contrast, another study reported that PET imaging could detect peritoneal metastatic lesions of a mucinous adenocarcinoma of the appendix because the FDG uptake was influenced by mitotic activity, tumor grade, Ki 67 index, and so on [15]. In our case, an invisible appendix with large cystic masses in the RLQ of the abdomen prevented an accurate diagnosis on the preoperative PET/CT examination. Extensive multiple seeding nodules in the whole abdomen, including the greater omentum, and associated with a moderate number of ascites were believed to be the aggravation of peritoneal disseminations of a mucinous neoplasm. In addition, no enlarged lymph nodes were seen in the peritoneum or retroperitoneum.

Miettinen et al. [5] reported the clinicopathological features of 95 cases of GISTs presenting with omental masses. Although they did not confine their report to pure omental GISTs and included some tumors attached to the gastrointestinal tract, the 95 cases were divided into solitary omental GISTs and multiple omental GISTs. The positivity rates of KIT, CD34, and SMA were $93 \%, 61 \%$, and $32 \%$, respectively, in solitary omental GISTs and were $95 \%, 33 \%$, and $14 \%$, respectively, in multiple omental GISTs. Immunohistochemical staining of our case revealed positive signals for c-KIT and negative signals for CD34 and SMA. In the survival analysis of the Miettinen et al. [5] 's study the median survival for multiple omental GISTs was reported to be 8 or 9 months, showing a poorer prognosis than solitary omental GISTs. We should mention that the patients in the above study did not receive any kind of imatinib treatment. The clinical impact of using adjuvant imatinib to treat multiple omental GISTs requires further investigation.

In conclusion, an EGIST is an uncommon disease and is difficult to diagnose by imaging alone. However, this disease might be 
included as one of the differential diagnoses of patients with multiple cystic lesions and seeding nodules along the mesentery and peritoneum and with an unknown primary tumor.

\section{CONFLICT OF INTEREST}

No potential conflict of interest relevant to this article was reported.

\section{REFERENCES}

1. Laurini JA, Carter JE. Gastrointestinal stromal tumors: a review of the literature. Arch Pathol Lab Med 2010;134:134-41.

2. Miettinen M, Lasota J. Gastrointestinal stromal tumors: pathology and prognosis at different sites. Semin Diagn Pathol 2006;23: 70-83.

3. Fletcher CD, Berman JJ, Corless C, Gorstein F, Lasota J, Longley BJ, et al. Diagnosis of gastrointestinal stromal tumors: a consensus approach. Hum Pathol 2002;33:459-65.

4. Kindblom LG, Remotti HE, Aldenborg F, Meis-Kindblom JM. Gastrointestinal pacemaker cell tumor (GIPACT): gastrointestinal stromal tumors show phenotypic characteristics of the interstitial cells of Cajal. Am J Pathol 1998;152:1259-69.

5. Miettinen M, Sobin LH, Lasota J. Gastrointestinal stromal tumors presenting as omental masses--a clinicopathologic analysis of 95 cases. Am J Surg Pathol 2009;33:1267-75.

6. Wong CS, Chu YC, Khong PL. Unusual features of gastrointestinal stromal tumor on PET/CT and CT imaging. Clin Nucl Med 2011;36:e1-7.

7. Burkill GJ, Badran M, Al-Muderis O, Meirion Thomas J, Judson IR, Fisher C, et al. Malignant gastrointestinal stromal tumor: dis- tribution, imaging features, and pattern of metastatic spread. Radiology 2003;226:527-32.

8. Kim HC, Lee JM, Kim SH, Kim KW, Lee M, Kim YJ, et al. Primary gastrointestinal stromal tumors in the omentum and mesentery: CT findings and pathologic correlations. AJR Am J Roentgenol 2004;182:1463-7.

9. Choi H. Imaging modalities of gastrointestinal stromal tumors. J Surg Oncol 2011;104:907-14.

10. Zaknun JJ, Kendler D, Moncayo R, zur Nedden D, Virgolini I. F-18 FDG PET for assessing tyrosine kinase-related signal transduction inhibition in a GIST c-kit-positive tumor patient by imatinib. Clin Nucl Med 2005;30:749-51.

11. Banzo I, Quirce R, Martinez-Rodriguez I, Jimenez-Bonilla J, Sainz-Esteban A, Barragan J, et al. F-18 FDG PET/CT assessment of gastrointestinal stromal tumor response to sunitinib malate therapy. Clin Nucl Med 2008;33:211-2.

12. Kamiyama Y, Aihara R, Nakabayashi T, Mochiki E, Asao T, Kuwano $\mathrm{H}$, et al. 18F-fluorodeoxyglucose positron emission tomography: useful technique for predicting malignant potential of gastrointestinal stromal tumors. World J Surg 2005;29:1429-35.

13. Berger KL, Nicholson SA, Dehdashti F, Siegel BA. FDG PET evaluation of mucinous neoplasms: correlation of FDG uptake with histopathologic features. AJR Am J Roentgenol 2000;174:1005-8.

14. Rohani P, Scotti SD, Shen P, Stewart JH, Russell GB, Cromer M, et al. Use of FDG-PET imaging for patients with disseminated cancer of the appendix. Am Surg 2010;76:1338-44.

15. Basu S, Shet T. FDG avid "abdominal band" representing omental cake in mucinous adenocarcinoma of the appendix: potential implications for disease monitoring with FDG-PET in this setting. J Cancer Res Ther 2011;7:231-3. 